\title{
TREE DETECTION USING UAV DATA AND DEEP LEARNING METHOD: FIRST STEP TOWARDS GREEN CADASTRE IN ESKIŞEHIR TECHNICAL UNIVERSITY CAMPUS
}

\author{
DOI: https://doi.org/10.18509/AGB.2020.22
}

UDC: $528.44: 630 * 1]: 004.8 / .9(560)$

\section{Gordana Kaplan, Nuri Erkin Öçer, Fırat Erdem, Dilek Küçük Matcı, Uğur Avdan}

\author{
Earth and Space Sciences Institute, Eskisehir Technical University, Eskisehir, Turkey
}

Corresponding author: kaplangorde@gmail.com

submitted: 13.03 .2020

accepted: 31.05 .2020

published: 15.09 .2020

\begin{abstract}
With the rapid urbanization and industrialization, people become more aware of the value of the green spaces. Green cadastre is an optimal solution to protect and preserve green areas in both urban and rural areas as it contributes to the proper maintenance of all green areas including trees and shrubs. In the last few years, the idea of green cadastre has been approved by many European cities and some have started conventional measurements for that purpose.

As a first step towards green cadastre in the Eskisehir Technical University campus, in this study, we use remote sensing and geo-information systems (GIS) techniques for tree detection and creating a GIS database. For this purpose, we apply a deep learning method namely mask region-based - convolutional neural network (mask R-CNN) for automatic tree detection using high-resolution data from Unmanned Arial Vehicle (UAV). Afterward, several GIS techniques have been used for extracting the attributes of the detected trees.

The results showed that the accuracy assessment of the applied methodology is higher than $90 \%$ which gives researchers the courage to use remote sensing data and GIS techniques for collecting data needed in the green cadastre database. The results of this study can be encouraging for starting green cadastre in many cities. For future studies we recommend applying the same methodology to other study areas, using additional data for achieving higher accuracy, and investigation for automatic classification of different tree types
\end{abstract}

Keywords: Green Cadastre, Remote Sensing, Tree detection, Convolutional Neural Network, Unmanned Arial Vehicle

\section{INTRODUCTION}

With the rapid urbanization and industrialization, people become more aware of the value of the green spaces. Green cadaster is an as optimal solution to protect and preserve green areas in both urban and rural areas as it contributes to the proper maintenance of all green areas including trees and shrubs. Although the idea of green cadaster is older, in 2014 the International Federation of Surveyors (FIG) pointed out that specific focus is needed for developing and operationalizing the concepts of green cadaster [1]. Namely, in 2014 FIG was searching for answers to the following question related to green cadaster: How can the ecological boundaries of green property rights be adjudicated, surveyed, and recorded? Do cadasters have a role? [1]. In the last few years, the idea of green cadaster has been approved and implemented by many European cities. For example, since 2011 the city of Skopje has been working on creating a GeoInformation Systems (GIS) database for the trees and shrubs in the capital of North Macedonia. The data collected with conventional measurements are available through a GIS web portal [2]. According to the results for the measured 650 ha, up-to-date, in the database are recorded 68.802 trees and 28.395 shrubs [3]. Poland [4] and Romania [5] have also made some efforts toward green cadaster. A successful green cadaster GIS database for trees is the one for the city of Zagreb [6], and for all green areas, the one for the City of Beograd [7]. Although current databases are well structured, the use of conventional data collection is time-consuming 
which makes it hard to maintain and to keep it updated. In the past few years, there is rapid development in the object-counting remote sensing based methodology. While at first researches were using object-based classification techniques for object extraction [8], nowadays researchers are exploring the opportunity of deep learning techniques and methods [9]. Thus, several studies have reported high success of deep learning methods for tree extraction and tree counting using remote sensing imagery [10-14]. Provided appropriate labeled data for training, this method enables machines to automatically learn representations from data such as images without introducing hand-crafted rules or human domain knowledge. Region-based Convolutional Neural Networks (R-CNN) is a state-of-the-art visual object detection system that combines bottom-up

\section{METHODS}

\section{Study Area and Data}

Located in the Anatolian region, in just $200 \mathrm{~km}$ from the capital, Ankara, Eskisehir which is wellknown as a city of science, culture, and youth, is home to three of the biggest universities in Turkey. As a study area in this paper Eskisehir Technical University, 2 Eylul Campus has been selected. Eskisehir Technical University is home to approximately 12.000 students and 1.300 staff members. For now, the active part of the 2 Eylul Campus is approximately 93 ha, with large green areas, a high number of trees and shrubs. The location and the study area of this paper are shown in Figure 1. The flight planning is one of the most region proposals with rich features computed by a convolutional neural network. The model used in this study, an extension of the R-CNN algorithm, Mask R-CNN [15] was released in 2018 and has become one of the most powerful algorithms for object detection and instance segmentation. Taking into consideration the disadvantage of the conventional measurements methods for green cadaster, and the trending in the remote sensing community, as a first step towards automatic green cadaster, in this study we employed Mask R-CNN for tree extracting from Unmanned Aerial Vehicle (UAV) data that later will be used for creating and GIS database for the trees in the study area. As a study area in this paper, Eskisehir Technical University, 2 Eylul Campus, Eskisehir Turkey has been selected.

important stages of UAV data acquisition. For the purpose of this study, the flight was performed on 23 September 2018 over the Eskisehir Technical University campus. The lateral overlap of the images was set to be $65 \%$, and the longitudinal overlap was set to be $75 \%$. The flight planning was made in the eMotion3 sesnseFly software. The acquired data were post-processed in Pix4D software. Details about the flight plan are given in Table 1. As a result of the data acquisition, a 4.2 $\mathrm{cm} / \mathrm{px}$ image with three channels (RGB) of the campus has been processed.

Table 1. Flight planning details

\begin{tabular}{ll}
\hline UAV & SenseFly eBee Plus \\
Camera & Sensefly S.O.D.A (RGB) \\
Lateral overlap & $65 \%$ \\
Longitudinal overlap & $70 \%$ \\
Altitude & $170 \mathrm{~m}$ \\
Planning Ground Sample Distance & $4.00 \mathrm{~cm} / \mathrm{px}$ \\
\hline
\end{tabular}




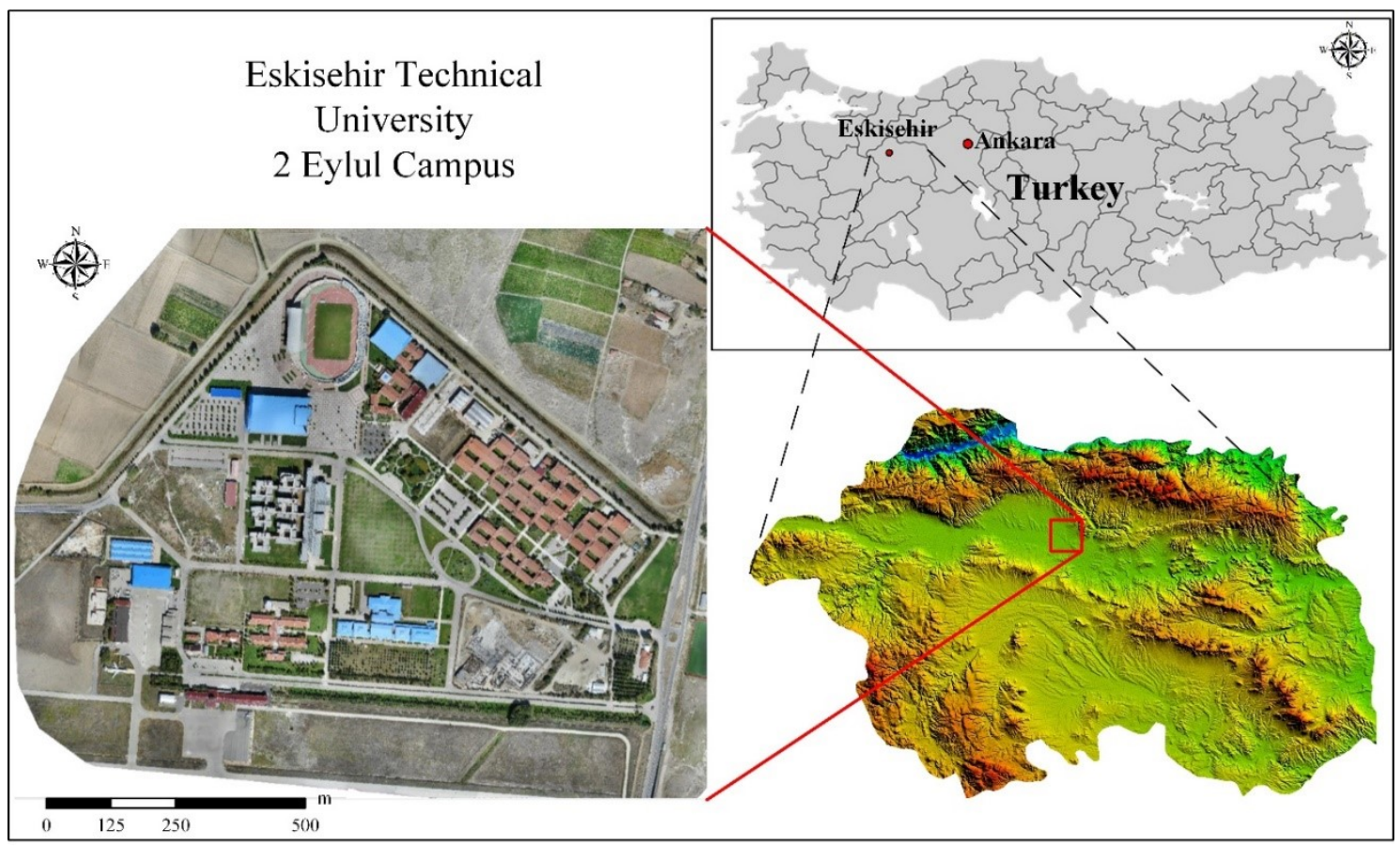

Figure 1. Study area, Eskisehir Technical University.

\section{METHODOLOGY}

As a first step towards green cadaster in the Eskisehir Technical University campus, in this study we use remote sensing and geo-information systems (GIS) techniques for tree detection and creating a GIS database, we apply a deep learning method namely mask region-based - convolutional neural network (mask R-CNN) for automatic tree detection using high-resolution data from Unmanned Arial Vehicle (UAV). Afterward, several GIS techniques have been used for extracting the attributes of the detected trees. The framework of the model used in this study is shown in Figure 2. It consists of four components: A backbone network architecture, bottom-up pathway, top-down pathway, and predictor's heads. In this study, Resnet-50 has been employed as the backbone network and its weights are initialized using a pre-trained network on Common Objects in Context (COCO) dataset

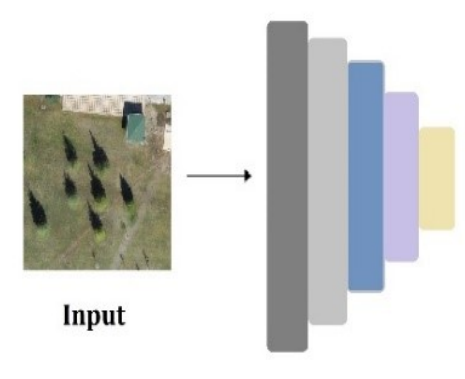

Backbone Network

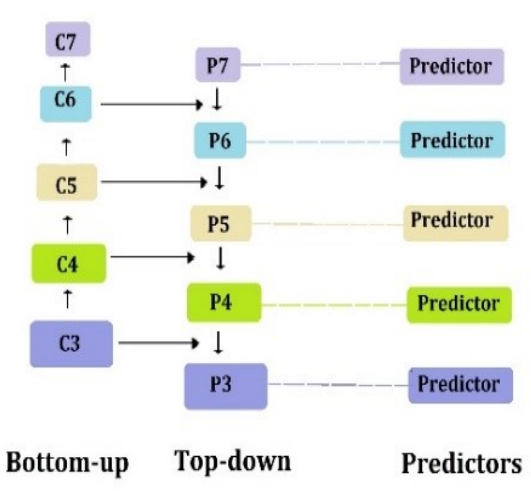

Figure 2. Framework model.

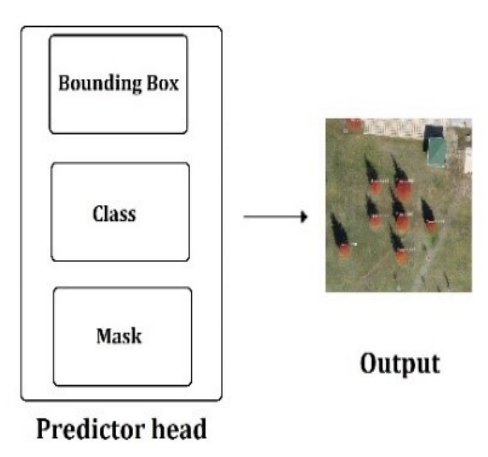

Predictor head
The implementation used in this study is based on the framework introduced by [17] which uses Keras and Tensorflow libraries. Data augmentation is used in order to increase training samples. Random scaling, rotation, vertical/horizontal flipping, contrast/brightness alteration, and Gaussian blurring are used. This technique helps to increase the amount of training data which prevents the model from over-fit and thus boosting the performance. In addition, it is helpful in training 
bimodels with small datasets such as the one implemented in this study. The model has been evaluated on a custom dataset. It consists of 352 annotated RGB images with $4 \mathrm{~cm} /$ pixel, where each image contains at least one tree. Image size is $768 \times 768$ pixels and there are several types of trees in the scenes. Trees in the images were annotated manually with masks as ground-truth.

$$
\begin{aligned}
& \text { precision }=\frac{T P}{T P+F P} \\
& \text { recall }=\frac{T P}{T P+F N} \\
& F=2 \cdot \frac{\text { precision recall }}{\text { precision+recall }}
\end{aligned}
$$

Among this set, 256 images were used for training (\%73), 71 images for validation $(\% 20)$, and 25

\section{RESULTS}

For our Mask R-CNN model with FPN and a backbone of Resnet 50, it took about 3 seconds to process 25 test images (about 8.05 iterations per second). The test scene and the resulting binary mask map for trees are shown in Figure 3. In this images for testing $(\% 7)$. In order to evaluate the performance of the model on our data set, precision, recall and F1 score - an overall measure of a model's accuracy that combines precision and recall, were calculate where, TP stands for true positives, FP for false positives, and FN for false negatives. Detections are accepted as true positive if the predicted mask overlaps with the ground-truth otherwise it counted as false positive. False negatives correspond to overlooked trees by the model. After the detection of the trees, a database has been created and the attributes such as coordinates and tree height were filed using different geospatial analysis.

figure, 25 test images combined together to form a single $3840 \times 3840$ pixels $(25 \times 768 \times 768$ pixels $)$ scene. Figure 3 shows the generated tree masks in the test area.

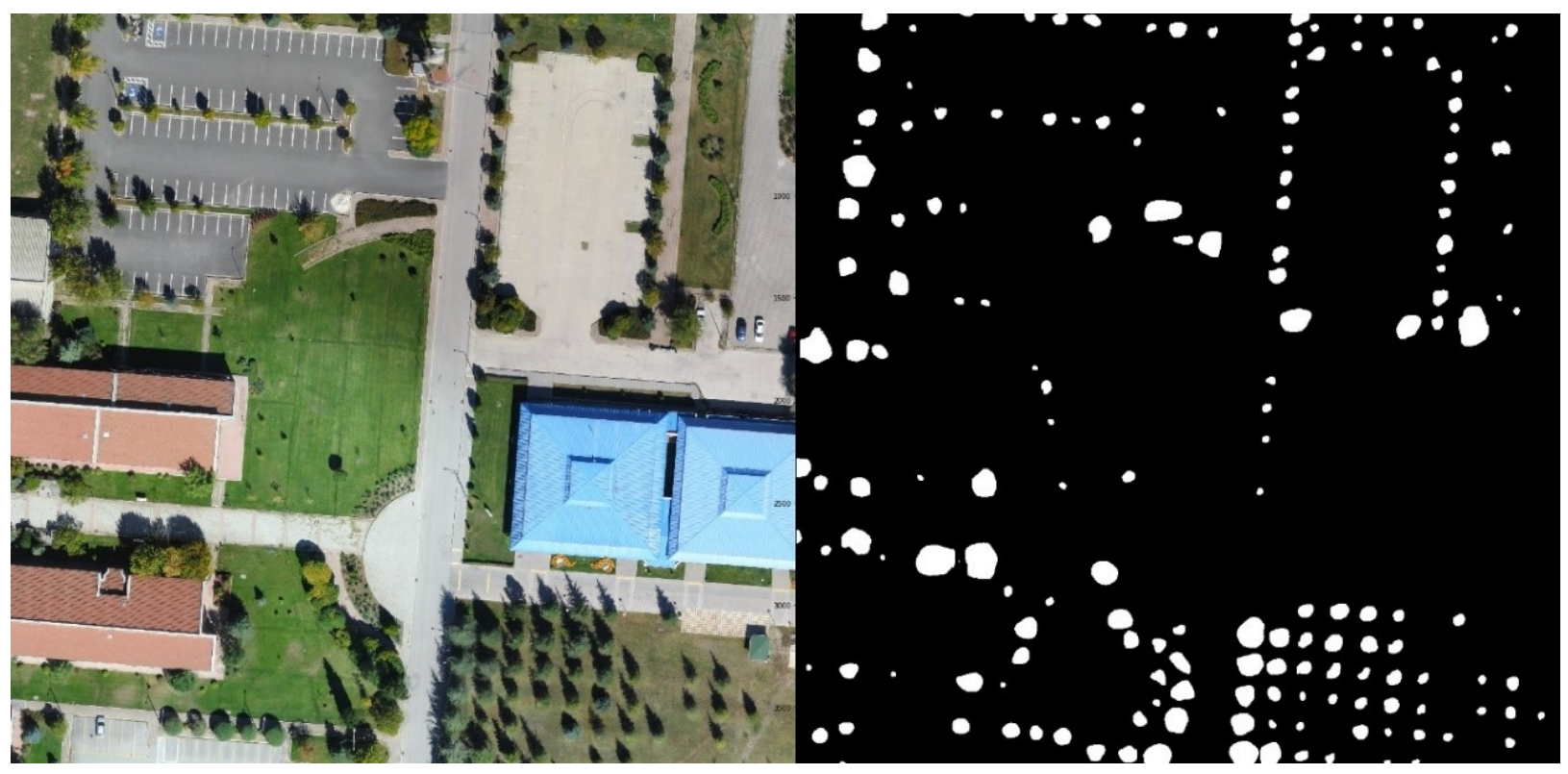

Figure 3. Test scene (left) and the resulting binary mask map for trees (right).

In the test region, the used model has detected 158 trees (missing 15 ground-truths) and generated 18 false alarms. It achieved a precision of 0.90 , a recall of 0.91 , and an F1 score of 0.91 . The results are shown in Figure 4 where the missing ground-truths are shown with a blue rectangle. In total, 3034 trees were detected in the study area. For every tree, the $\mathrm{X}, \mathrm{Y}, \mathrm{Z}$ coordinates were extracted. Since the terrain is flat, the approximate tree heights were extracted from the DTM of the study area with subtraction from the mean terrain value. 


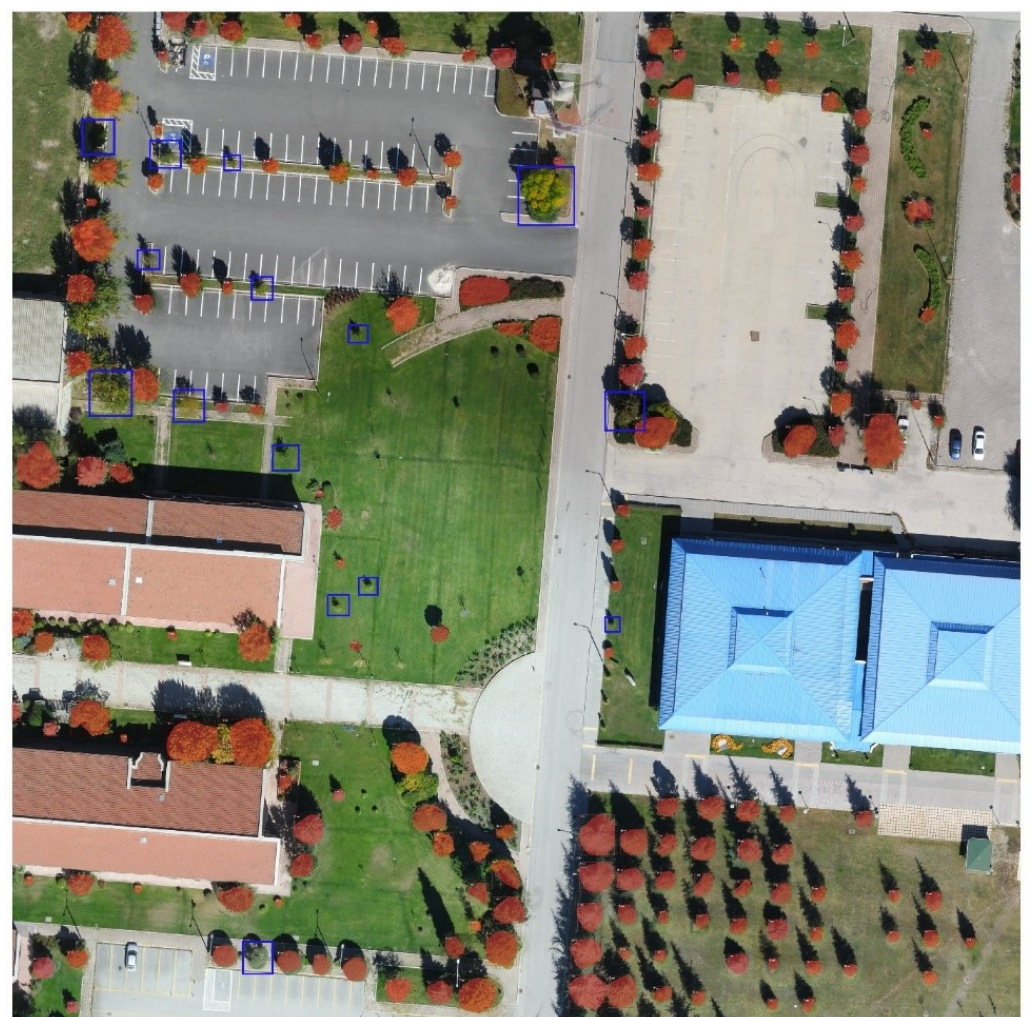

Figure 4. Results: detected trees - red; ground-truths missing - blue rectangles.

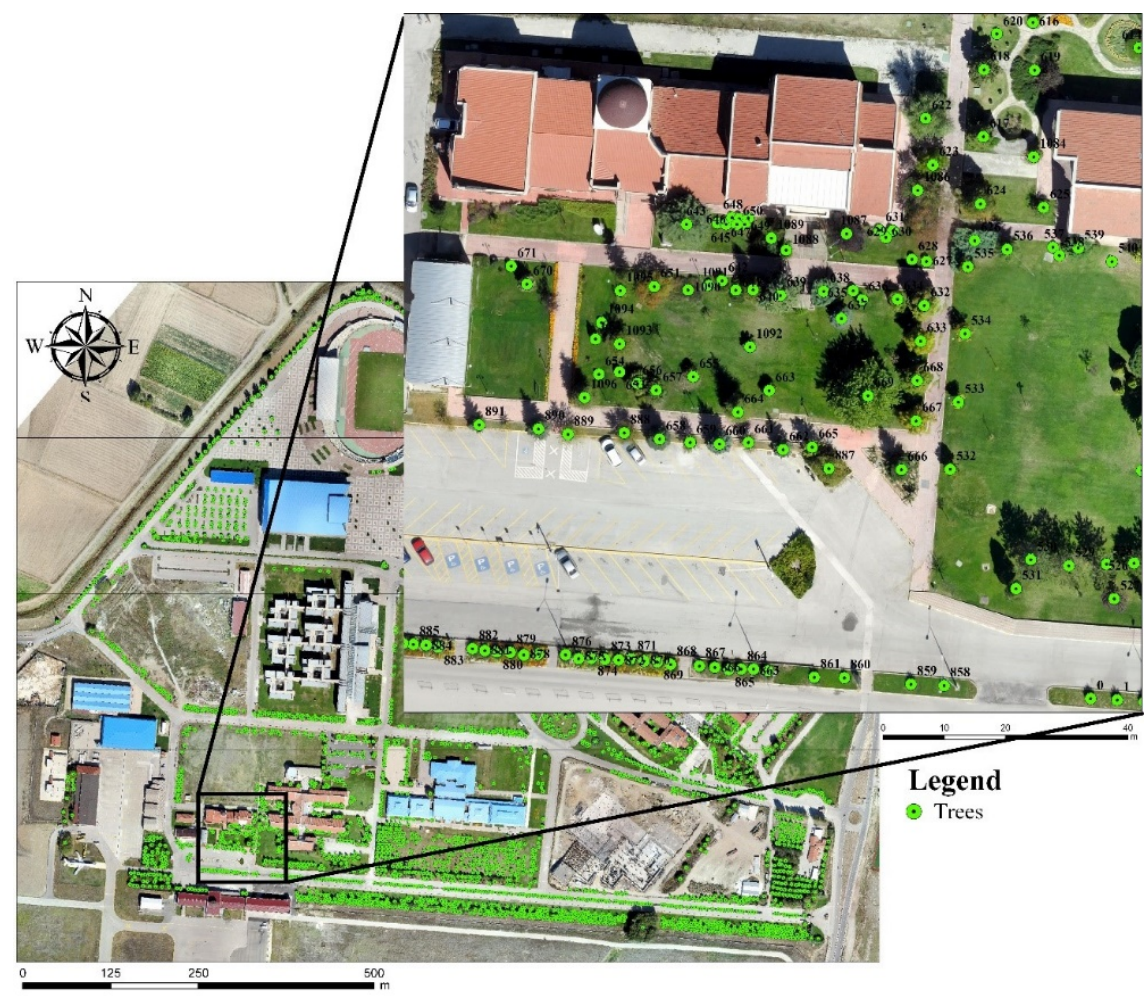

Figure 5. Results - ESTU_Tree map.

\section{DISCUSSION}

Green spaces contribute to environmental improvement, ecological balance, and harmonization between artificial and natural landscapes. The main objective of green cadaster is a contribution to good maintenance of the green structure of the environment. This paper presents a first step towards automated green cadaster in the Eskisehir Technical University, 2 Eylul Campus. There are several advantages and disadvantages between the conventional measurement methods 
and the method (Mask RCNN) and data (UAV) used in this study. One of the biggest advantages of the used method is that its less time consuming and practical. While with the conventional methods it would take weeks to collect the data, the model in this study takes less than one hour to produce the final results. However, the method used in this study is usually used for detecting one type of tree. In order to classify the trees according to their types, further analyses are required. Taking into consideration that only RGB data was used in this study, the use of additional data can be useful in

\section{CONCLUSION}

Green cadastre is an optimal solution to protect and preserve green areas in both urban and rural areas. As a first step towards green cadastre in the Eskisehir Technical University campus, in this study, we used remote sensing and GIS techniques for tree detection and creating a GIS database. For this purpose, we applied mask R-CNN deep learning method for automatic tree detection using high-resolution UAV data. The results showed that the accuracy assessment of the applied detecting different tree types. With additional analyses, we believe that the accuracy can be improved. Also, with additional geospatial analyses on the UAV data, the total green area on the campus can be extracted, as well as the total canopy area. Taking into consideration the overall results of this study, we believe that with further analyses, the UAV data and the Mask R-CNN method can be used, not only for the first step toward green cadaster but also for complete automated creating and maintaining the greed cadaster GIS database.

methodology is higher than $90 \%$ which gives researchers the courage to use remote sensing data and GIS techniques for collecting data needed in the green cadastre database. The results of this study can be encouraging for starting green cadastre in many cities. For future studies we recommend applying the same methodology to other study areas, using additional data for achieving higher accuracy, and investigation for automatic classification of different tree types.

\section{REFERENCES}

[1] Steudler, D., CADASTRE 2014 and Beyond. 2014: International Federation of Surveyors (FIG).

[2] Skopje, G., http://gis.skopje.gov.mk/zk/. 2019. (Accessed on 15.11.2019)

[3] Skopje,E., http://gdi-sk.maps.arcgis.com/ (Accessed 15.11.2019)

[4] Zysk, E., et al., ORGANIZATIONAL ASPECTS OF THE CONCEPT OF A GREEN CADASTRE FOR RURAL AREAS. Land Use Policy, 2019: p. 104373.

[5] Marian, M. and A.N. Matei, Aspects of green cadastre in the campus of University of Pitesti. EDITORIAL BOARD, 2012: p. 52.

[6] Zagreb, G., https://gis.zrinjevac.hr/. 2019. (Accessed on 18.11.2019)

[7] Beograd, G., http://gispublic.zelenilo.rs/giszppublic/Map. 2019. (Accessed on 18.11.2019)

[8] Kaplan, G. and U. Avdan, Object-based water body extraction model using Sentinel-2 satellite imagery. European Journal of Remote Sensing, 2017. 50(1): p. 137-143.

[9] Zhang, L., L. Zhang, and B. Du, Deep learning for remote sensing data: A technical tutorial on the state of the art. IEEE Geoscience and Remote Sensing Magazine, 2016. 4(2): p. 22-40.

[10] Li, W., et al., Deep learning based oil palm tree detection and counting for high-resolution remote sensing images. Remote Sensing, 2017. 9(1): p. 22.

[11] Mubin, N.A., et al., Young and mature oil palm tree detection and counting using convolutional neural network deep learning method. International Journal of Remote Sensing, 2019. 40(19): p. 7500-7515.

[12] Ribera, J., et al. Counting plants using deep learning. in 2017 IEEE global conference on signal and information processing (GlobalSIP). 2017. IEEE.

[13] Xia, M., et al. Fast and robust detection of oil palm trees using high-resolution remote sensing images. in Automatic Target Recognition XXIX. 2019. International Society for Optics and Photonics. 
[14] Weinstein, B.G., et al., Individual tree-crown detection in RGB imagery using semi-supervised deep learning neural networks. Remote Sensing, 2019. 11(11): p. 1309.

[15] He, K., et al. Mask r-cnn. in Proceedings of the IEEE international conference on computer vision. 2017.

[16] Lin, T.-Y., et al. Microsoft coco: Common objects in context. in European conference on computer vision. 2014. Springer.

[17] https://github.com/matterport/Mask_RCNN. (Accessed on 22.12.2019) 